\title{
15 años de la revista Persona
}

David Álvarez Flores

Universidad de Lima

Este año la Universidad de Lima cumple cincuenta años de creación inspirada en la búsqueda de la verdad científica y la formación de profesionales altamente capacitados, con cultura humanística y criterios de permanente actualización y superación. Durante los años 1960 y 1961, un grupo de profesores universitarios e importantes representantes del comercio y la industria, reunidos en la Asociación Civil Promoción del Desarrollo Industrial a través de la Educación Superior (Prodies), decidió fundar una nueva universidad. Después de dos años de esfuerzo conjunto se logró su creación. Prodies se planteó como objetivo la creación de una nueva casa de estudios diferente, en la que se fomentara el espíritu comunitario entre profesores y alumnos. La Universidad inició sus labores con 120 alumnos, agrupados en dos facultades. Debido a su rápido crecimiento, en agosto de 1966 se inauguró el campus de Monterrico y, posteriormente, en 1987 el campus de Mayorazgo.

Actualmente, la Universidad cuenta con tres Escuelas Universitarias que administran un total de once carreras, una Escuela de Postgrado y un Programa de Estudios Generales; además cuenta con un Centro de Educación Continua, un Instituto de Investigación, y servicios para los estudiantes y la comunidad universitaria en general. La población es de aproximadamente 15.000 alumnos, 1000 docentes y 400 trabajadores administrativos. Tiene dos campus universitarios, el de Monterrico, con un área construida de $142.143 \mathrm{~m}^{2}$, y el campus de Mayorazgo, con $30.000 \mathrm{~m}^{2}$. Ambos centros presentan moderna infraestructura y tecnología de vanguardia a fin de contribuir con el logro de los propósitos establecidos.

El contexto de la formación de psicólogos en la década de 1990 estaba conformado por ocho universidades, todas ellas ubicadas en la capital. Estas eran: Universidad Nacional Mayor de San Marcos, Universidad Femenina del Sagrado Corazón, Universidad Inca Garcilaso de la Vega, Universidad Federico Villarreal, Universidad Ricardo Palma, Universidad San Martín de Porres, Universidad Cayetano Heredia y Pontificia Universidad Católica del Perú. Según el tipo de gestión, dos eran estatales y seis privadas.

Correo electrónico: dalvarez@ulima.edu.pe 
En 1992 se crea la Facultad de Ciencias Humanas, con las especialidades de Psicología e Historia; de esta manera, se inicia la formación de psicólogos en la Universidad de Lima. La profesión se fortalece en el año 1994, con la creación de la Facultad de Psicología, obteniendo así autonomía académica.

Desde sus inicios la Facultad de Psicología tiene como misión formar psicólogos con una sólida base teórica y metodológica de la ciencia básica y aplicada, con una actitud amplia, tolerante y antidogmática frente a los cambios científicos y sociales; ofreciendo una formación profesional general y flexible, mediante el otorgamiento del título profesional de licenciado en Psicología, situación que permite al egresado insertarse con una mayor facilidad en el mercado laboral.

La carrera recoge en su currículo los avances actuales de la psicología como teoría y práctica científicas; asimismo, cuenta con un laboratorio de primer nivel compuesto por un gabinete psicométrico, una sala de experimentos, salas de observación individual y grupal y sala de grabación. Además, el programa de prácticas se realiza en diferentes centros laborales y se complementa con el apoyo a la comunidad mediante dos proyectos sociales: Programa de Educación Básica Laboral (PEBAL) y Viña Alta, los cuales van involucrando al alumno en el compromiso social, como un rasgo distintivo de la carrera.
Entre las fortalezas de la Facultad de Psicología se puede mencionar a los docentes, la titulación, las investigaciones y los niveles de satisfacción de los estudiantes, los egresados y los empleadores. Así, se cuenta con un plantel docente que ostenta el grado de doctor $(19 \%)$ y de maestría (28\%), porcentajes que están por encima de los estándares de calidad exigidos. Se cuenta con medio millar de titulados y alta tasa de titulación de acuerdo con los estándares de calidad. Las investigaciones tienen dos fuentes: las auspiciadas por el Instituto de Investigación de la Universidad de Lima y las que son producto de las tesis para la obtención del título profesional. Gran parte de las investigaciones se orientan a la adaptación, validación y normalización de pruebas psicológicas, constituyendo el aporte de nuevos instrumentos para el diagnóstico e intervención profesional en diferentes poblaciones.

Otra fortaleza que merece ser destacada es el nivel de satisfacción en relación con la formación académica, el plan curricular, la plana docente, los servicios académicos y los servicios administrativos, la infraestructura y las tecnologías de la información. Igualmente, los empleadores se encuentran satisfechos con las competencias que poseen nuestros egresados contratados por sus respectivas organizaciones.

\section{La revista Persona}

Los avances de la psicología como ciencia y profesión, el número de institucio- 
nes formadoras de profesionales en la especialidad, los escasos espacios para difundir los resultados de las investigaciones y los planteamientos de los aportes teóricos llevaron a un conjunto de profesores de la Facultad de Psicología a emprender esfuerzos conjuntos para la creación de una revista que sirva como punto de encuentro de una serie de posiciones teóricas y profesionales. Estos esfuerzos rindieron sus frutos, $\mathrm{y}$ en 1998, coincidiendo con el egreso de la primera promoción, sale a la luz el primer número de la revista Persona.

Desde su aparición, el objetivo que orientó la creación de Persona fue constituirse en un foro amplio, crítico y plural, donde se consideran las contribuciones teóricas y de carácter metodológico, reportes de investigación, revisiones de literatura y reflexiones acerca de temas de interés de la psicología como generadora de conocimientos e intervención profesional, con la finalidad de que puedan ser dados a conocer para la discusión y la posible réplica, que son rasgos distintivos de la actividad académica y científica. Se ha buscado incluir contribuciones que traten acerca de las nuevas especialidades que van surgiendo con el avance científico y tecnológico, así como las 56 divisiones que considera la Asociación Psicológica Americana (APA). Otro de sus objetivos fue constituirse en una revista de alta calidad académica, teniendo como referente a las tradicionales revistas de psicología de nivel internacional. Debe mencionarse que en el año 2011, el Colegio de Psicólogos del Perú otorgó el "Reconocimiento de la Orden a la Revista Persona, en mérito al esfuerzo sostenido y a los estándares de calidad alcanzados, convirtiéndola en un medio de divulgación de investigaciones a nivel latinoamericano".

Entre las principales características que progresivamente le han permitido alcanzar logros relevantes están las siguientes:

a) $\mathrm{Su}$ publicación ininterrumpida, a lo largo de quince años, en un contexto donde se permite y acepta la cultura de lo temporal.

b) El comité editorial, cuyos signos distintivos son el reconocido nivel académico y la continuada permanencia de sus miembros a lo largo de su existencia editorial, que le ha otorgado el estilo característico a la revista.

c) El hecho de contar con un Comité Científico Nacional y un Comité Científico Internacional, integrado por distinguidas personalidades del mundo académico, que han fortalecido su continuidad.

d) La intervención de expertos, que han aportado su experiencia como evaluadores de pares imprimiéndole la exigencia académica.

e) La participación de un conjunto de autores de los países de Iberoamérica y Europa, que ha permitido la permanencia de esta revista. 
El Comité Científico Nacional de la revista Persona está integrado por académicos de la Universidad Nacional Mayor de San Marcos (cinco), la Universidad Cayetano Heredia, la Universidad Ricardo Palma, la Pontificia Universidad Católica del Perú, la Universidad de San Martín de Porres, la Universidad Femenina Sagrado Corazón, la Universidad de Lima, el Instituto Nacional de Salud Mental Hideyo Noguchi-Honorio Delgado, la Consultora Adapta y el Instituto de Investigación de Marketing Arellano.

Por su parte, el Comité Científico Internacional acoge a académicos de Argentina (tres), Colombia (dos), Chile, Estados Unidos de América, Alemania, Bélgica, España, Portugal y de la Organización Internacional del Trabajo (OIT).

\section{NúMEROS PUBLICADOS Y AUTORES}

Sin pretender realizar un estudio bibliométrico en profundidad, haremos un balance de los números publicados desde 1998 hasta el 2011. Para cumplir con este objetivo se procedió a elaborar una base de datos de todos los artículos publicados durante ese periodo, la cual contiene: autor, nacionalidad, título, número de autores participantes en la publicación, tipo de artículo (teórico/ empírico), enfoque, área de aplicación y metodología empleada en los reportes de investigación (alcance de la investigación, diseño, participantes e instrumentos).
Así, podemos mencionar que en dicho lapso se han publicado 14 números, con un total de 125 artículos, 39 reseñas y 19 resúmenes de tesis; en promedio se ha incluido 9 artículos por número, de los cuales el 59\% corresponde a autores nacionales, $35 \%$ a autores extranjeros y $6 \%$ a contribuciones compartidas entre autores nacionales y extranjeros. En la producción de artículos han intervenido 194 autores, $56 \%$ peruanos y $44 \%$ de extranjeros. En cuanto al número de autores participantes en la elaboración de los artículos se encuentra que $70 \%$ corresponde a un solo autor, $16 \%$ a dos autores y $14 \%$ a más de tres autores. Estos dos últimos casos, casi un tercio de la producción, representan un avance que favorece el trabajo en equipos, tal como lo exige la investigación en el presente momento.

Entre los autores destacados por su productividad en la revista se encuentran, con 7 artículos Ricardo Braun (filosofía de la ciencia) y Miguel Escurra (psicometría e investigación educacional); con 5 artículos Nelly Ugarriza (psicometría); con 4 artículos Jozef Corveleyn (psicoterapia, emociones y apego), Ramón León (psicología social), Manuel Fernández (psicología educacional) y Alejandro Parra (percepción extrasensorial), y con 3 artículos Willy Lens (motivación), Ana Delgado (psicometría), Raúl González Moreyra (psicología cognitiva), Marta Gil Lacruz (psicología social), David Álvarez (psicología educacional) y Natalia Torres (psicoanálisis). 


\section{TIPO DE ARTÍCULOS}

Teniendo en cuenta el tipo de artículos, los reportes de investigaciones empíricas ascienden al 59\% y $41 \%$ al enfoque teórico.

En relación con los 49 artículos de corte teórico se encuentran varias vertientes en esta producción: las revisiones teóricas corresponden al 35\%, relacionados con la historia de la psicología al $16 \%$, conectados con la filosofía de la psicología al 18\%, y ensayos al 31\%. En cuanto a la temática, se encuentra una variedad, como la motivación, los desórdenes psicológicos, la relación terapéutica, el psicoanálisis, la bioética, el psicodiagnóstico de Rorschach, entre otros.

En lo que se refiere al campo aplicativo de los artículos, 44\% pertenecen a la psicología educativa, $37 \%$ a la clínica y de la salud, 7\% a la organizacional y $7 \%$ a la psicología social.

Según la temática de los artículos de corte empírico, se aprecia que el interés de los autores se orienta de manera mayoritaria hacia la psicometría $(23,3 \%)$, la misma que se dirige a la adaptación, la determinación de la validez y la confiabilidad, el desarrollo de normas de administración, y la calificación e interpretación de los resultados de instrumentos psicológicos. La gama de pruebas psicológicas estudiadas aborda especialmente las de inteligencia y personalidad. Este trabajo constituye un notable aporte a la profesión en la medi- da en que se puede contar con pruebas psicológicas adaptadas y normalizadas a nuestra realidad.

\section{Metodología}

En relación con la metodología empleada en los estudios empíricos se aprecia que casi la totalidad de las investigaciones (94\%) corresponde a las de tipo transversal y solo $6 \%$ al longitudinal. Según el alcance del proceso de investigación se encuentra que 30\% pueden incluirse en el tipo descriptivo, $67 \%$ al correlacional y $3 \%$ al experimental. El diseño predominante es el comparativo.

Teniendo en cuenta el número de participantes que han intervenido en los reportes de investigación, se puede mencionar que $23 \%$ corresponde a muestras menores a 100 participantes, $46 \%$ a muestras entre 100 y 500 participantes y $31 \%$ a muestras entre 500 y 5000. En cuanto a la edad de los participantes, cobran preponderancia las muestras en las cuales solo participan adolescentes (51\%), le siguen los adolescentes y adultos (19\%), los solo adultos $(16 \%)$, los niños y adolescentes (3\%) y los exclusivamente niños (11\%). En cuanto a las poblaciones estudiadas, las muestras consideran a hombres y mujeres, planteamiento que permite realizar las comparaciones, indicadas anteriormente. Los reportes de investigación señalan que el tipo de participantes seleccionados está integrado por 
muestras de personas normales (82\%), pacientes $(5 \%)$ y normales y pacientes (12\%), para permitir realizar la contrastación de resultados.

En relación con el nivel de educación de las muestras, la mayor parte de investigaciones corresponden a estudiantes universitarios (37\%), a los de nivel secundario el $36 \%$, a los de educación superior el $9 \%$, a los de primaria el 15\% y a los de educación preescolar el 3\%.

Los instrumentos utilizados son variados: muestran preponderancia las escalas de actitudes (35\%) y los cuestionarios (32\%); le siguen los inventarios (11\%) y los programas de intervención (5\%).

\section{INTERNACIONALIZACIÓN}

La premisa acerca de que el conocimiento que no se difunde no existe condujo a la necesidad de ampliar su difusión a entornos globales, esto es, la internacionalización de la revista Persona. Para cumplir con tal objetivo se realizaron las gestiones para que sea incluida en diferentes bases de datos especializadas. Actualmente, la revista se encuentra indizada en siete bases de datos: Sistema Regional de Información en Línea para Revistas Científicas de América Latina (Latindex), Servicios de índices electrónicos de la Universidad de Rioja, España, para revistas e información (Dialnet), Base de datos bibliográficos especializados en temas afines al Colegio Oficial de Psicólogos de Madrid
(PsicoDoc), Colección de revistas científicas electrónicas en Psicología y áreas afines de la Biblioteca Virtual en SaludPsicología y la Asociación Brasileña de Editores Científicos de Psicología (PePSIC), Red de Revistas Científicas de América Latina y el Caribe, España y Portugal (Redalyc), Organización de Estados Iberoamericanos (OEI) y la Base de datos bibliográficos de revistas de ciencias sociales y humanidades (Clase). La presencia en estas bases de datos y el estar a disposición del público en general ha permitido su amplia difusión.

\section{Perspectivas}

En la perspectiva de lograr la mejora continua de la revista, nos planteamos algunos objetivos que serán logrados con la intensiva participación de los comités científicos, nacional e internacional, y el comité editorial. Uno de ellos es contar con un mayor número de contribuciones de carácter internacional para mantener los estándares internacionales. Otro objetivo, a corto plazo, es conseguir la incorporación de Persona en otras bases de datos para compartir la producción del conocimiento.

Asimismo, consideramos que es necesario ampliar el espectro de las áreas temáticas de los artículos orientándose a las nuevas corrientes científicas, especialmente, a los enfoques multidisciplinarios. Igualmente, propender a la publicación de reportes de investigación 
elaborados por equipos interdisciplinarios y cuyas poblaciones de estudio provengan de metodologías y poblaciones diversas.

Un objetivo permanente del equipo editorial es lograr la periodicidad semestral y la publicación de números monográficos, hecho que solo podrá lograrse con la dirección del comité editorial y el aporte de los autores.

La publicación no hubiera podido llegar al lugar que ocupa actualmente de no ser por el invalorable aporte de los profesionales del Fondo Editorial, quienes han brindado su experiencia y exigencia en la tarea de cumplir con los objetivos propuestos por Persona.

Finalmente, reafirmamos el compromiso de seguir publicando una revista del más alto nivel académico, en aras de favorecer el intercambio de artículos para el logro de la internacionalización que la ubique al alcance de la sociedad. 